\title{
Disputas en torno al carácter de la educación pública en Uruguay: de la privatización latente a la privatización impuesta
}

\section{Disputas sobre o caráter da educação pública no Uruguai: da privatização latente à privatização imposta}

\section{Disputes over the character of public education in Uruguay: from latent privatization to imposed privatization}

\author{
Pablo Martinis*
}

\begin{abstract}
RESUMEN
El artículo aborda, desde una perspectiva vinculada al análisis político del discurso, los procesos de privatización educativa en desarrollo en Uruguay en la última década. A través del análisis de materiales documentales se muestra como durante los gobiernos de centro izquierda del Frente Amplio (2005 - 2019) se generaron condiciones para una incipiente privatización. Se analiza que un primer paso dado por los sectores favorables a la privatización fue el de generar en la opinión pública una visión fuertemente crítica de la educación pública uruguaya. Estos sectores, integrados por actores empresarios, políticos y académicos, hicieron uso de la modalidad de "donaciones especiales" para financiar proyectos educativos definidos como "públicos de gestión privada". Estos proyectos educativos fueron presentados como alternativas frente a la educación pública. A través de la información analizada se muestra como estas iniciativas han ganado espacios
\end{abstract}

* Universidad de la República. Montevidéu, Uruguay. E-mail: pablomartinis@gmail.com - https://orcid.org/0000-0002-1210-0098 
en la ciudadanía uruguaya. Finalmente se presenta como estas propuestas son retomadas y profundizadas por el gobierno de derechas instalado el 1 de marzo de 2020. Se deja abierta la necesidad de continuar profundizando en investigaciones sobre estos temas a los efectos de comprender de mejor forma los procesos en curso.

Palabras claves: Educación. Privatización. Gestión. Discurso. Uruguay.

\title{
RESUMO
}

$\mathrm{O}$ artigo aborda, a partir de uma perspectiva vinculada à análise política do discurso, os processos de privatização educacional em desenvolvimento no Uruguai na última década. Por meio da análise de material documental, mostra-se como durante os governos de centro-esquerda da Frente Ampla (2005 - 2019) se geraram condições para uma privatização incipiente. Analisa-se que um primeiro passo dos setores partidários da privatização foi gerar na opinião pública uma visão fortemente crítica da educação pública uruguaia. Esses setores, compostos por atores empresariais, políticos e acadêmicos, utilizaram a modalidade "doações especiais" para financiar projetos educacionais definidos como "públicos privados". Esses projetos educacionais foram apresentados como alternativas à educação pública. As informações analisadas mostram como essas iniciativas têm ganhado espaço entre os cidadãos uruguaios. Por fim, apresenta-se como essas propostas são retomadas e aprofundadas pelo governo de direita instalado em $1^{\circ}$ de março de 2020. Fica em aberto a necessidade de continuar a aprofundar as pesquisas sobre esses temas para melhor compreender os processos em andamento.

Palavras-chave: Educação. Privatização. Gestão. Discurso. Uruguai.

\begin{abstract}
The article addresses, from a perspective linked to the political analysis of discourse, the processes of educational privatization in processes in Uruguay in the last decade. Analysis of documentary materials shows how during the left-center governments of the Frente Amplio (2005 - 2019), conditions were created for a fledgling privatization. It is analyzed that a first step taken by the pro-privatization sectors was to generate in public opinion a strongly critical view of the Uruguayan public education. These sectors, made up of entrepreneurial, political and academic actors, used the "special donations" modality to finance educational projects defined as "privately run public educational projects". These educational projects were presented as alternatives to public education. Through the analyzed information, it is shown how these initiatives have gained space in Uruguayan citizens. Finally, this initiative is presented as being taken up and deepened by the
\end{abstract}


right-wing government installed on March 1, 2020. The need to continue deepening research on these issues is left open in order to better understand the ongoing processes.

Keywords: Education. Privatization. Management. Discourse. Uruguay.

\section{Presentación}

El presenta artículo aborda los procesos de privatización educativa en desarrollo en Uruguay. Se trabaja desde una perspectiva teórica afectada por el análisis político de discurso (LACLAU; MOUFFE, 2004) y una estrategia metodológica basada en análisis de fuentes documentales.

El texto se organiza en cuatro apartados. En el primero se explicita la perspectiva teórica y metodológica desde la que se elabora el trabajo de investigación presentado. En la segunda, se expone como a través de la utilización de la modalidad de "donaciones especiales", un mecanismo para facilitar a través de renuncias fiscales donaciones a diversos tipos de instituciones, se construyó una coalición empresaria, académica y política para la promoción de una "educación pública de gestión privada". También se enfatiza acerca de cómo esta forma de organización de la educación con fuerte presencia de actores privados fue presentada como alternativa a la educación pública. En el tercer apartado se analizan los modos a través de los cuales el nuevo gobierno de derechas instalado a partir del 1 de marzo de 2020 ha continuado los procesos iniciados previamente, a los efectos de profundizar los niveles de privatización en la educación uruguaya. Finalmente, se sintetizan los aportes volcados en el artículo y se señala la necesidad de continuar ampliando los procesos de de investigación sobre la temática, asumiendo el carácter indefinible de lo social y su apertura a diversos escenarios de futuro.

\section{Enfoque conceptual y abordaje metodológico}

El enfoque teórico general que nutre los procesos de investigación en los que se enmarca el presente texto reconoce una afectación de la del análisis político del discurso (LACLAU; MOUFFE, 2004). De esta perspectiva tomamos particularmente la noción de "imposibilidad de la sociedad" (LACLAU, 1993), 
la cual nos ubica en un campo de problematización de lo social que supone una perspectiva filosófica antiesencialista y antifundacionalista. Esto implica,

\begin{abstract}
Aceptar la infinitud de lo social, es decir, el hecho de que todo sistema estructural es limitado, que está siempre rodeado por un "exceso de sentido" que el es incapaz de dominar y que, en consecuencia, la "sociedad" como objeto unitario c inteligible que funda sus procesos parciales, es una imposibilidad (LACLAU, 1993, p. 104).
\end{abstract}

Se renuncia a la existencia de un momento fundacional en el cual la sociedad habría adquirido sentidos últimos e inmanentes, asumiendo el carácter histórico y sobredeterminado de los procesos sociales. De aquí se desprende que las identidades sociales tampoco pueden ser definidas en términos fijos e inmutables, ya que no se asume la existencia de un factor externo que las dote de coherencia y sentido absoluto. Asumir esta posición teórica implica reconocer que toda identidad se constituye en términos relacionales, en tanto que,

Si mantenemos el carácter relacional de toda identidad y si, al mismo tiempo, renunciamos a la fijación de esas identidades en un sistema, en ese caso lo social debe ser identificado con el juego infinito de las diferencias, es decir, con lo que en el sentido más estricto del término podemos llamar discurso -a condición, desde luego, de que liberemos al concepto de discurso de un significado que lo restringe al habla y a la escritura (LACLAU, 1993, p. 104-105).

Concebir lo social en términos de "infinito juego de diferencias" supone un movimiento conceptual de interés para nuestro trabajo, ya que permite tomar nota de la permanente disputa que la construcción de órdenes sociales supone entre posiciones ideológicas antagónicas.

Entender la sociedad como una constante disputa entre posiciones diferentes no implica dejar de reconocer que estas disputas se dirimen en el marco de relaciones de fuerza que se asientan en la base material de esa sociedad. No desconocemos las relaciones de fuerza inherentes a las relaciones sociales, tan solo descartamos que éstas determinen los resultados de esas disputas. Adherimos a la idea de que las bases materiales de una sociedad condicionan las posibilidades de constitución de dicha estructura, pero no asumimos que 
determinen los resultados finales de las disputas existentes. Partimos de una noción de historia como construcción basada en disputas permanentes entre fuerzas antagónicas, disputas cuyos resultados no pueden necesariamente ser establecidos a priori.

En línea con lo que venimos desarrollando nos parece relevante rescatar la distinción entre lo político y la política que introduce Chantal Mouffe (2009). En palabras de la propia autora:

Concibo "lo político" como la dimensión del antagonismo que considero constitutiva de las sociedades humanas, mientras que entiendo a "la política" como el conjunto de prácticas e instituciones a través de las cuales se crea un determinado orden, organizando la coexistencia humana en el contexto de la conflictividad derivada de lo político (MOUFFE, 2009, p. 16).

La distinción que plantea Mouffe resulta de utilidad analítica en la medida en que nos permite apreciar a lo político como el máximo nivel de abstracción, ubicando al conflicto como elemento constitutivo de las relaciones sociales. El conflicto supone una dimensión constitutiva de las sociedades humanas, la cual toma forma en las disputas por la construcción de posiciones hegemónicas en el marco de lo que la autora define como la política. La disputa concreta en la arena política tiene que ver con el intento permanente de crear y tornar válidas diversas formas de organizar la sociedad y las interacciones entre sus actores. Esta disputa mantiene permanentemente abierta la posibilidad de cambios y de construcción de nuevos órdenes sociales, en tanto la sociedad como espacio en última instancia "imposible" no se constituye ni se organiza nunca en forma plena.

Una visión crítica de las relaciones sociales debería integrar el reconocimiento del carácter inherentemente conflictivo de lo social, así como la imposibilidad de una resolución racional y definitiva de las disputas. Se trata de asumir lo social como espacio de conflicto permanente e insertar en esa perspectiva las luchas por la construcción de órdenes más democráticos, asumiendo que ninguna ley de la sociedad puede ser referida para pretender justificar de modo ontológico y finalista la primacía de un orden. Ningún destino ni social está trazado de antemano, la construcción de futuros deseables supone la intervención en la esfera política.

Desde aquí, sostenemos como hipótesis que es probable que una visión no del todo clara de estos fenómenos haya permeado a las fuerzas progresistas 
a nivel global en las últimas décadas. Asumimos que la creciente asunción de perspectivas liberales sobre lo social por parte de las fuerzas progresistas ha atentado contra su comprensión de la política como un espacio de disputas inerradicables. Esta es la hipótesis acerca de la imposición de un horizonte pospolítico en la concpeción de las relaciones sociales que introduce Mouffe. Ello implica un problema en términos de avance democrático, por la existencia de actores "pertenecientes al campo progresista, (que) aceptan esta visión optimista de la globalización, y han pasado a ser los defensores de una forma consensual de democracia" (MOUFFE, 2009, p. 9).

La asunción de la posibilidad de construir consensualmente la democracia por parte de sectores progresistas está en la base de los avances de sectores de matriz neoliberal y conservadora. Estos han logrado avanzar con la imposición de sus diagnósticos y sus alternativas. Como señala Mouffe, esta reticencia a asumir el carácter inherentemente conflictivo de lo social entrampa a los sectores progresistas en una visión del mundo en la que las categorías de derecha e izquierda pierden fuerza interpretativa, quedando ocultas las disputas hegemónicas. De este modo la dimensión del antagonismo es sustituido por la suposición liberal de que es posible construir ordenes consensuales donde todos podríamos vernos reflejados por igual.

Desde las claves teóricas que aquí hemos apenas esbozado, es posible desarrollar una lectura que explica los avances que los procesos de privatización de la educación han tenido en gobiernos que se definen como progresistas. Una clave que distingue la instalación de un orden pospolítico en los discursos educativos tiene que ver con la instalación de la idea de que los problemas educativos pueden ser abordados a través de intervenciones técnicas (BORDOLI; ROMANO, 2009). Estas serían llevadas adelante por actores que se desenvuelven desde perspectivas racionalmente instrumentales, dejando de lado toda referencia al carácter político, por tanto conflictivo, de los problemas educativos. Un elemento asociado a este tiene que con la asunción a nivel global de la idea de que las soluciones de mercado y la privatización constituyen claves para superar los problemas de la educación pública (ADRIÃO; PERONI, 2008; PERONI, 2013; BALL, 2014, BELLEI; ORELLANA, 2014; VERGER; FONTDEVILA; ZANCAJO, 2016).

Ambas definiciones, la de la educación como problema técnico y la de la carácter deseable de una educación organizada sobre la base de procesos pro mercado, han permeado el pensamiento progresista. Si bien la temática es de interés en todo el ámbito latinoamericano, en el presente artículo analizaremos exclusivamente el caso uruguayo.

Nuestra lectura de los procesos de privatización se nutrirá de la distinción establecida por Ball y Youdell (2008) entre privatización exógena y endógena. 
En el primer caso, se hace referencia a procesos que implican la expansión y creciente influencia del sector educativo privado en el conjunto de un sistema de educación y el establecimiento de alianzas público - privado. En cuanto a la privatización endógena, supone la asunción de principios del ámbito privado en la educación pública. Para la lectura del caso uruguayo interesan ambas perspectivas, ya que es posible apreciar la existencia de ambas, con una primacía de la privatización endógena.

La exposición sobre el caso uruguayo que se presentará en los próximos apartados se organiza a partir de una lectura de dos procesos históricos. Por una parte, procesos desarrollados en los años en los que la coalición progresista Frente Amplio estuvo en el gobierno (2005 - 2019). En segunda instancia propondremos una lectura de una etapa hoy en curso, marcada por la asunción del gobierno por parte de una coalición de derechas encabezada por el candidato del Partido Nacional, actual presidente, Luis Lacalle Pou. Esta etapa se presenta como de profundización y generalización de acciones pro privatización iniciadas anteriormente.

A la primera de las etapas mencionadas la identificaremos con la noción de "privatización latente" (VERGER; MOSCHETTI; FONTDEVILA, 2017). Estos autores sostienen que Uruguay, país reconocido por el peso hegemónico de la educación pública, ha ingresado en las primeras décadas del siglo XXI en procesos velados y paulatinos de privatización.

A la segunda de las etapas mencionadas, actualmente en curso, la definiremos desde la noción de privatización impuesta, mediante la cual buscamos dar cuenta de iniciativas a partir de las cuales el nuevo gobierno pretende profundizar procesos de privatización educativa.

Desee el punto de vista de la estrategia metodológica empleada, hemos analizado documentos gubernamentales, estudiado a las empresas que realizan donaciones e indagado acerca de vinculaciones de las mismas con ámbitos de poder económico y político, revisado el contenido de sitios webs de diversas instituciones privadas que impulsan procesos de privatización y analizado el contenido de un proyecto de ley mediante el cual el nuevo gobierno uruguayo pretende generar transformaciones en la educación. La metodología utilizada toma la propuesta de Ball (2011, p. 25) de una "etnografía política", en tanto "mapeo de la forma y contenido de las relaciones de la política en un campo particular". 


\section{Generación de condiciones para promover la "educación pública de gestión privada"}

\section{La modalidad de "donaciones especiales"}

Uno de los componentes que alimentó la expansión de los postulados privatizadores en Uruguay fue el uso que un sector del empresariado, aliado con otros actores, hizo del instrumento de las "donaciones especiales" (BORDOLI et al., 2017; MOSCHETTI et al., 2019). Este instrumento se introdujo en la legislación en la reforma tributaria de 2007 (URUGUAY, 2007), la cual habilita que empresas privadas realicen donaciones pudiendo descontar impositivamente el $81,25 \%$ de lo donado. El marco ha sido luego perfeccionado a través de diversas normativas ${ }^{1}$.

Se trata de un instrumento que permite el redireccionamiento de recursos estatales por parte de empresas privadas. El procedimiento ha sido utilizado para promover a instituciones educativas privadas que serán presentadas como alternativas a la educación pública.

La siguiente tabla nos muestra los montos donados entre los años 2010 y 2018.

\section{TABLA 1. MONTO DE DONACIONES ESPECIALES RECIBIDAS POR} INSTITUCIONES EDUCATIVAS EN EL PERÍODO 2010 - $2018^{2}$.

\begin{tabular}{|c|c|c|c|c|c|c|c|c|c|}
\hline \multicolumn{10}{|c|}{$\begin{array}{l}\text { DONACIONES RECIBIDAS 2010-2018 (Parte I) } \\
\text { En millones de pesos a valores corrientes }\end{array}$} \\
\hline AREA & 2010 & 2011 & 2012 & 2013 & 2014 & 2015 & 2016 & 2017 & 2018 \\
\hline Educación Primaria, Secundaria y Técnico Profesional & 31,8 & 45,4 & 29,0 & 53,0 & 108,9 & 118,4 & 115,8 & 130,5 & 155,9 \\
\hline ANEP & 31,3 & 27,0 & 4,8 & 4,7 & 8,5 & 13,1 & 9,1 & 7,5 & 7,2 \\
\hline Liceo Jubilar & 0,5 & 6,3 & 7,6 & 11,8 & 13,4 & 15,0 & 16,2 & 12,0 & 18,9 \\
\hline Colegio Ma. Auxiliadora & - & - & - & 1,0 & 0,0 & - & - & - & - \\
\hline Fundación impulso & - & 12,1 & 16,6 & 35,5 & 66,2 & 64,0 & 58,0 & 68,6 & 73,1 \\
\hline Liceo Providencia & . & . & & . & 11,3 & 11,0 & 12,0 & 13,0 & 15,0 \\
\hline Liceo Francisco & - & - & - & . & 9,4 & 9,1 & 10,0 & 10,8 & 11,5 \\
\hline Colegio Sagrado Corazón & - & - & - & - & - & 2,9 & 2,5 & 3,1 & 2,7 \\
\hline Bachillerato Tec. Ánima-Tec & - & - & - & - & - & 3,5 & 8,0 & 8,6 & 9,2 \\
\hline Liceo San José de Tala & - & - & - & - & - & - & - & 0,8 & 0,8 \\
\hline Fundación Sophia & - & - & - & . & - & - & - & 6,2 & 12,5 \\
\hline Los Rosales & - & - & - & - & - & - & - & - & 5,0 \\
\hline
\end{tabular}

FUENTE: Tomado de: MEF (2018).

1 Las leyes 18834 (2012), 19149 (2014), 19438 (2018) y 1967 (2018), introdujeron ajustes de forma en el procedimiento. Todas las leyes referidas están disponibles para su consulta en www. parlamento.gub.uy

2 La sigla ANEP refiere a la Administración Nacional de Educación Pública, entre autónomo responsable de toda la educación básica pública nacional. Las demás instituciones presentes en la tabla pertenecen al ámbito privado. 
Durante los dos primeros años de vigencia del mecanismo de donaciones especiales gran parte de los recursos donados lo fueron a la educación pública. Esta situación comenzó a alterarse a partir del año 2012. Basta como muestra que ilustre sobre este proceso observar los dos extremos de los años referidos. En el año 2010, de cada 100 pesos donados, 98,4 llegaron a la educación pública. En el año 2018, de cada 100 pesos donados, 4,6 llegaron a ese destino. Si a este dato le sumamos el hecho que las donaciones totales se incrementaron un $490 \%$ entre el año 2010 y el 2018, resulta sencillo apreciar que la movilización de recursos hacia el sector privado fue muy significativa. Como datos claves para comprender el crecimiento real de los recursos donados, tómese nota que, según datos del Ministerio de Economía y Finanzas de Uruguay, en el período 2010 - 2018 el índice de precios al consumo se incrementó en un 188,62\% y el valor del dólar se incrementó en un 63\% (MARTINIS, 2019). Se trata de un incremento muy significativo de fondos, los cuales son absorbidos casi exclusivamente por el sector privado. Esto debe leerse como un proceso exitoso de redireccionamiento de fondos públicos hacia instituciones privadas.

En el presente trabajo haremos referencia a una de las instituciones beneficiarias por ser la que ocupa el lugar central en la estrategia presentar a la educación "pública de gestión privada" como alternativa superadora de la educación pública. Se trata del caso del Liceo Impulso.

Esta institución educativa privada de enseñanza media está ubicada en el barrio más empobrecido de la ciudad de Montevideo. Fue fundada con el propósito explícito de implementar un proyecto educativo exitoso para la superación de la "brecha de aprendizajes" existente entre los adolescentes más ricos y los más pobres de Uruguay ${ }^{3}$.

\section{El caso del Liceo Impulso}

Martinis (2019) ha planteado características del Liceo Impulso que lo colocan en un lugar privilegiado entre las instituciones que perciben donaciones especiales. En el presente trabajo ubicaremos algunos de esos ejes centrales a los efectos de dar profundizar en dicha caracterización. Los núcleos a los que nos interesa referirnos son: a) origen y justificación del Liceo Impulso; b) recursos captados por esta institución; c) función ideológica que cumple la existencia del liceo. Luego de abordar estos tres puntos, nos detendremos en dos aspectos que

3 Toda la información sobre el Liceo Impulso está tomada de: http://www.liceoimpulso. edu.uy/fundacin 
consideramos sustantivos en la estrategia analítica que define el presente artículo: características de los principales donantes de Impulso y uso de la experiencia de esta institución en el diseño de la estrategia de privatización educativa que ha delineado el nuevo gobierno de derechas instalado en Uruguay en marzo de 2020.

\section{a) Origen y justificación del Liceo Impulso.}

La institución desde la cual se genera la propuesta es la Fundación Impulso. Allí convergen un conjunto de actores claves del pensamiento neoliberal en Uruguay. Se trata de: Ernesto Talvi ${ }^{4}$, Pablo Da Siveira ${ }^{5}$, Nicolás Herrera ${ }^{6}$, Elbio Strauch $^{7}$, Marcelo Guadalupe y Horacio Hughes.

Según se señala en su web, es un liceo "de tiempo completo, laico, gratuito y de gestión privada en una zona de contexto crítico para procurar contribuir a eliminar la brecha de aprendizaje"».

A través de esta somera declaración, es posible dar cuenta de las principales características y finalidad de la institución.

Por una parte, se menciona el carácter laico y gratuito de la institución. Con estos significantes se está haciendo referencia directa al modo de definir la educación que está inscrito en la tradición pedagógica uruguaya desde el último tercio de siglo XIX. Desde entonces, la educación formal en Uruguay se reconoce como "laica", "gratuita" y "obligatoria". Es muy clara la intencionalidad manifiesta de entroncar la existencia del Liceo Impulso en esa tradición uruguaya.

No obstante, debe señalarse que esta caracterización deja de lado un elemento sumamente importante y que da sentido a los anteriores: la posibilidad en la educación uruguaya de acceder a un nivel educativo si se han aprobado los anteriores. En el Liceo Impulso ese acceso no está previsto, ya que según las reglamentaciones que posee, solamente podrán aspirar a matricularse en la institución estudiantes mayores de 14 años al momento de la inscripción ${ }^{9}$. Por tanto, si bien el acceso es gratuito, no es libre. Resulta claro que quienes quedan

4 Economista (Universidad de la República) y Doctorado en Economía (Universidad de Chicago). Director del Centro de Estudios de la Realidad Económica y Social (CERES), uno de los principales think tanks neoliberales de Uruguay (https://ceres-uy.org). Desde el 1 de marzo de 2020 es Ministro de Relaciones Exteriores.

5 Licenciado en Filosofía (Universidad de la República) y Doctor en Filosofía (Universidad de Lovaina). Académico. Ministro de Educación y Cultura del gobierno instalado el 1 de marzo de 2020 .

6 Abogado con extensa trayectoria. Integra el Consejo Directivo del CERES y es miembro de la Mont Pelerin Society. CV detallado disponible en https://www.guyer.com.uy/es/professionals/ herrera-nicolas/

7 Strauch, Guadalupe y Hughes son destacados actores de ámbitos empresarios uruguayos.

8 Cita tomada de: http://www.liceoimpulso.edu.uy/fundacin

9 Ver requisitos para la inscripción en el 2020: http://www.liceoimpulso.edu.uy/inscripciones 
fuera de la posibilidad de matricularse en la institución son aquellos estudiantes con mayores dificultades en el aprendizaje.

En segundo lugar es importante tomar nota de la construcción del significante "gratuito y de gestión privada". Las instituciones que perciben financiamiento por "donaciones especiales" se han autodefinido como "públicas de gestión privada", en tanto no cobran matrícula a sus alumnos. Es posible apreciar que en el armado de la frase la noción de "gratuito" ocupa el lugar de la significación "público". Se opera de este modo una reducción de la potencialidad significativa de la noción de lo "público" al reducirla exclusivamente el carácter gratuito de la institución. Si a esto agregamos el hecho de que no todos los potenciales estudiantes con interés de asistir al Liceo Impulso pueden hacerlo, es posible notar que la significación democrática y republicana asociada a la noción de "educación pública" queda sumamente afectada.

Finalmente, es necesario hacer referencia a la apelación a "contribuir a eliminar la brecha de aprendizaje", que se presenta en la definición del sentido de la institución. Este es uno de los principales elementos a partir de los cuales la institución pretende constituirse en una alternativa a la educación pública. En Uruguay existen resultados inequitativos en cuanto a lo culminación del ciclo educativo de enseñanza media entre estudiantes que ocupan posiciones extremas en la distribución de la riqueza. A partir de actuar sobre este problema es que el Liceo Impulso pretende justificar su existencia e incidir en el debate en torno a la caracterización de las relaciones entre público y privado en la educación uruguaya. Volveremos sobre tema más adelante.

\section{b) Recursos captados por el Liceo Impulso.}

Los recursos distribuidos por "donaciones especiales" tuvieron un fuerte crecimiento entre 2010 y 2018. Este crecimiento supera a la inflación y al costo del dólar. El Liceo Impulso, según surge del análisis, es el mayor beneficiario de estos incrementos.

Comienza a percibir "donaciones especiales" en el año 2011. Ese año, recibe 12,1 millones de pesos sobre un total de 45,4 donados $(26,65 \%$ del total). En el año 2018 recibió 73,1 millones de pesos sobre un total de 155,9 (46,89\% del total). En los demás años, el porcentaje recibido sobre el total de lo donado osciló entre un mínimo de 52,57\% en 2017 y un máximo de 66,98\% en 2013. Esta información muestra que la institución fue la elegida por los donantes como el emblema fundamental de la modalidad de "educación púbica de gestión privada".

Partiendo de esta constatación resulta particularmente interesante estudiar a las empresas que donan a Impulso. Presentaremos aquí algunos avances de una tarea en desarrollo en nuestro equipo. Tomamos el período 2013 - 2018 por ser este en el que se registran mayores donaciones. En la Tabla 2 presentamos 
la cantidad de empresas que donaron en cada año a Impulso. Las donaciones se dirigen a la Fundación Impulso, titular del Liceo.

TABLA 2. EMPRESAS DONANTES A FUNDACIÓN IMPULSO POR AÑOS SELECCIONADOS

\begin{tabular}{|c|c|c|c|c|c|}
\hline 2013 & 2014 & 2015 & 2016 & 2017 & 2018 \\
\hline 42 & 59 & 55 & 45 & 53 & 48 \\
\hline
\end{tabular}

FUENTE: Elaboración propia en base a datos de: $\operatorname{MEF}(2019,2018,2017,2016,2015,2014)$.

La tabla muestra una continuidad importante en número de empresas que donan a Impulso. Muchas empresas donan a lo largo de todo el período y otras lo hacen puntualmente.

Buscando caracterizar el núcleo fuerte de donantes de Impulso, nos detuvimos en observar a aquellos que en el período hubieran donado al menos un promedio de un millón de pesos uruguayos por año. Este recorte nos ubicó frente a un total de diecisiete empresas. Estas, tomadas en conjunto, explican un $65.65 \%{ }^{10}$ de las donaciones recibidas entre 2013 y 2018.

Entre las empresas que integran este grupo de grandes donantes, encontramos una amplitud de rubros comerciales: banca privada, producción de bebidas, industria frigorífica, crédito al consumo, industria del papel, construcción, supermercados, industria metalmecánica, entre otros. En un caso, un donante figura con su propio nombre y apellido en representación de otros más: Nicolás Herrera y Otros, ya referido entre los integrantes de la Fundación Impulso.

Actualmente nuestro trabajo de investigación está dedicado a indagar acerca de la posible existencia de redes que vinculen a los principales actores de estas empresas entre sí y con ámbitos de producción de perspectivas favorables a los procesos de privatización. Para la producción del presente artículo, hemos optado por realizar un corte todavía más especifico en la mirada de los donantes. Tomamos los cinco que figuran con mayores niveles de donación en el período analizado. La información sistematizada puede apreciarse en la Tabla 3.

10 Tomando los datos de los informes realizados por el Ministerio de Economía y Finanzas entre los años 2014 y 2019, se puede apreciar que las donaciones totales recibidas por la Fundación Impulso en el período fueron de 365.330.358 millones de pesos; en tanto que el aporte de las empresas seleccionadas fue de 250.806 .443 . 
TABLA 3. CINCO MAYORES DONANTES A FUNDACIÓN IMPULSO EN EL ACUMULADO DEL PERÍODO 2013 - 2018.

\begin{tabular}{|l|c|}
\hline Empresa donante & $\begin{array}{l}\text { Monto donado a Fundación Impulso en el } \\
\text { período 2013 - 2018 }\end{array}$ \\
\hline Nicolás Herrera y Otros & 48.239 .000 \\
\hline Tenaris Global Services S.A. & 29.121 .819 \\
\hline Enalur S.A. & 19.200 .000 \\
\hline Supermercados Disco del Uruguay S.A. & 16.563 .600 \\
\hline Fabricas Nacionales de Cerveza S.A. & 15.545 .756 \\
\hline
\end{tabular}

FUENTE: Elaboración propia en base a información contenida en MEF (2019, 2018, 2017, 2016, 2015, 2014).

Estos cinco donantes concentran el 35,22 de las donaciones habidas por Impulso en el período. Es interesante notar que quien encabeza la lista es el ya mencionado Nicolás Herrera.

Entre las otras empresas donantes, se ubica una que opera en el mercado del acero (Tenaris Global Services) con presencia en más de 30 países ${ }^{11}$. Uno de sus referentes es Paolo Rocca, una de las principales fortunas de la Argentina. Según informes de prensa, Rocca instala su empresa en Uruguay luego de enfrentamientos con el gobierno argentino en 2008, contando con el beneplácito de actores del gobernante Frente Amplio ${ }^{12}$. Según el mismo medio, la empresa sería objeto de investigaciones en el marco de la Operación Lava Jato en Brasil ${ }^{13}$.

En tercer lugar se ubica Enalur S.A., empresa de telecomunicaciones, subsidiaria de "Liberty Global"14, compañía multinacional con sede en Londres, Amsterdam y Denver ${ }^{15}$. Es proveedor del estado uruguayo ${ }^{16}$.

Por otra parte, se ubica un referente en el ámbito del supermercadismo: Supermercados Disco del Uruguay S.A. Es una empresa del "Grupo Cardoso", grupo económico que además de manejar varias cadenas de supermercados,

11 Información obtenida de https://www.bnamericas.com/es/perfil-empresa/tenaris-globalservices-sa

12 Información presentada en edición del semanario "Caras y caretas" 30/6/2018. Ver: https://www.carasycaretas.com.uy/la-telarana-de-techint-y-tenaris/

13 Información presentada en edición del semanario "Caras y caretas" 30/6/2018. Ver: https://www.carasycaretas.com.uy/la-telarana-de-techint-y-tenaris/

14 Según información consultada en https://www.sec.gov/Archives/edgar/ data/1316631/000095013405008832/d24879exv21w1.htm

15 Ver: https://www.libertyglobal.com

16 Ver: http://www.comprasestatales.gub.uy/consultas/detalle/id/23967 
tiene fuerte influencia en el mundo de las telecomunicaciones, ya que maneja un canal de televisión abierta y medios de prensa escrita ${ }^{17}$.

Finalmente, se ubica Fábricas Nacionales de Cerveza, empresa del sector de la bebida. Según se señala en su propio sitio web, forma parte de "AnheuserBusch InBev, que se conformó a través de diversas combinaciones en los últimos años con distintas empresas como Ambev, Interbrew, Anheuser Bush, grupo Modelo, SAB Miller, entre otras"18. Según el mismo sitio, su CEO es el brasileño Carlos Brito, com amplia trayectoria en el rubro. Algunos artículos periodísticos vinculan a Brito con Paulo Lemann ${ }^{19}$, también parte de la industria de la bebida y referente de la Fundación Lemann (Brasil). Esta fundación, es una de las principales agencias de promoción de procesos de privatización educativa en Brasil (CAETANO, 2018).

A través de este recorrido sumario por algunas características de las principales empresas donantes de la Fundación Impulso, hemos pretendido dar cuenta de la coalición empresaria que se configuró para orientar el destino de fondos públicos hacia iniciativas privadas. Nos interesa llamar la atención acerca de algunas características de estos donantes: a) uno de los referentes de la Fundación Impulso y personalidad ligada al pensamiento neoliberal en Uruguay, figura como el articulador de un conjunto de actores que constituyen el principal donante; b) tres de las cuatro empresas donantes son parte de amplios conglomerados transnacionales; c) varias de ellas han tenido diversas formas de contacto político con referentes gubernamentales en el período 2005 - 2019; d) es posible ubicar relaciones de actores claves de estas empresas con ámbitos dedicados a la promoción de procesos de privatización educativa en la región. Estas características deberán ser sometidas a análisis en la continuidad de nuestra investigación.

\section{c) Función ideológica que cumple la existencia del Liceo Impulso.}

Los elementos desarrollados hasta aquí permiten delinear algunas hipótesis en torno al lugar ideológico que la existencia del Liceo Impulso ha ocupado en la educación uruguaya.

En primer lugar, resulta necesario apreciar que la propia creación de la Fundación Impulso debe ser comprendida en el marco de una estrategia concertada entre actores de la derecha uruguaya. Esta estrategia persigue una

17 Ver: https://adiaria.com.uy/articulo/2017/12/informe-sobre-concentracion-en-uruguaydetalla-otras-actividades-empresariales-de-grandes-grupos-mediaticos/ y también https://brecha. com.uy/todos-para-uno/

18 Información tomada de: https://www.fnc.com.uy/site/about-us

19 Ver: https://www.portafolio.co/economia/finanzas/ARTICULO-MOVILESAMP-323546.html 
doble finalidad. Por una parte responsabilizar a la educación pública de la existencia de una "brecha de aprendizajes"; por otra, presentar la modalidad de educación pública de gestión privada como la alternativa que permitiría la superación de esa situación.

Con respecto a la primera línea señalada, la misma se enmarca en el trabajo desplegado durante los gobiernos del Frente Amplio a los efectos de generar un sentido común en la sociedad uruguaya proclive a aceptar que la educación se encontraría sumida en una grave crisis. Esta estrategia, que contó con un papel activo de diversos actores de la derecha política, social y económica, fue amplificada por los grandes medios de comunicación.

La segunda linea de trabajo se caracterizó por una estrategia basada en resaltar los resultados de aprendizajes satisfactorios del Liceo Impulso en comparación con instituciones educativas públicas. La presentación de las Memorias Anuales del centro educativo ${ }^{20}$ ha difundido estos logros. Estos han sido amplificados por medios de comunicación y actores políticos.

La exposición de los logros del Impulso no hace referencia a sus procedimientos de selección de alumnado, ni a la abundancia de recursos económicos que posee. Por los recursos obtenidos a través de donaciones, el Liceo Impulso maneja montos que al menos duplican los disponibles en la educación pública. Según se ha establecido en una reciente investigación: "Desde 2015 (primer año con que se cuenta información del balance en la rendición de cuentas), por cada peso por estudiante que gasta el CES ${ }^{21}$, el Impulso gastó \$2,63 en 2015; \$2,2 en 2016; y \$ 2 en 2017” (DUFRECHOU et al., 2019, p. 110).

Como vemos, es posible apreciar el despliegue de una estrategia que ha apuntado a disputar el carácter público de la educación en Uruguay a través de tres líneas convergentes de trabajo:

1. La creación de un sentido común que asuma la situación crítica de la educación uruguaya y responsabilice de la misma a la educación pública. Esto puede apreciarse como los procesos de "escandalización de la opinión pública" que refiere Steiner-Khamsi (2003) en relación al tratamiento de los medios de comunicación sobre los resultados de la educación pública.

2. La conformación de una Fundación como ámbito de concepción de un proyecto y de recaudación de fondos para sostenerlo. Esta Fundación utiliza la modalidad de donaciones especiales, articulándose para

20 Ver Memorias Anuales del período 2014 -2018. Disponible en: http://www.liceoimpulso. edu.uy/memoria-anual

21 La sigla hace referencia al Consejo de Enseñanza Secundaria, organismo responsable del nivel de educación secundaria básica y superior en Uruguay. 
cumplir sus objetivos con empresas que acompañan su estrategia, redirigiendo fondos públicos hacia la estrategia pro-privatización.

3. La propia instalación del Liceo Impulso, que será tomado como laboratorio a los efectos de mostrar las "bondades" de la educación pública de gestión de privada en detrimento de la educación pública.

La eficacia de las líneas de trabajo planteadas, alimentó propuestas de extensión de esta modalidad educativa a todo el país. Ello marca el momento en que la realidad de la privatización deja de poder ser caracterizada como "latente" y se va conformando como una realidad presente. El triunfo de una coalición de derechas en las elecciones nacionales de fines de 2019 inaugura una nueva etapa en este proceso. A ello nos referiremos a continuación.

\section{Acceso de las derechas al gobierno y comienzo de una nueva fase de privatización}

El 1 de marzo de 2020 se instaló en el gobierno de Uruguay una coalición de derechas luego de triunfar en las elecciones nacionales del año 2019. Esta coalición, integrada por cinco partidos, colocó en la Presidencia al Dr. Luis Lacalle Pou, candidato del Partido Nacional.

A los efectos de dar cuenta de los avances de los procesos privatizadores en Uruguay en este contexto, se hace necesario hacer referencia a dos procesos convergentes. El primero de ellos comenzó a desarrollarse a partir del año 2016. Desde ese año, diversos actores políticos comienzan a señalar a necesidad de instalar en el país un nuevo modelo de centro educativo de enseñanza media. Este modelo educativo es inspirado en la experiencia del Liceo Impulso.

Por otra parte, es necesario mostrar cómo ciertas medidas que propone el nuevo gobierno dan continuidad y generan condiciones para la profundización de procesos de privatización.

\section{a) La propuesta de los "136 liceos modelo" en todo el país}

Con la intención de proceder a la generalización del modelo de educación pública de gestión privada el Centro de Estudios de la Realidad Económica y Social (think tank neoliberal al que ya nos hemos referido), lanza la propuesta de generar en todo el país un conjunto de liceos basados en el "modelo Impulso". El Director Ejecutivo del CERES, Ernesto Talvi (ya referido como uno de los creadores de la Fundación Impulso), toma el tema y trabaja en la difusión de 
la idea $^{22}$. En el año 2018, Talvi se lanza a la actividad política, logrando se electo como candidato a la Presidencia de la República por el histórico Partido Colorado. Como efecto de este movimiento la propuesta de creación los liceos modelo ingresa al programa de gobierno del mencionado partido (PARTIDO COLORADO, 2019, p. 166).

Al no haber podido acceder al balotaje que definiría la elección del 2019, el partido de Talvi se integró la coalición de derecha ya referida. En dicho marco se consensuó un programa de gobierno en el cual vuelve a encontrarse la propuesta de establecer los liceos modelo ${ }^{23}$.

Luego de este breve resumen de historia reciente de Uuguay, estamos en condiciones de apreciar el largo camino transitado por una propuesta de privatización educativa. Se ha ido desde una privatización latente hacia una posibilidad de privatización generalizada. Sobre las propuestas para la expansión del fenómeno privatizador trabajaremos en el próximo apartado.

\section{b) El fomento a los procesos de privatización educativa en el nuevo gobierno.}

El nuevo gobierno uruguayo ha basado su estrategia de introducción de cambios profundos en diversos ámbitos de la realidad nacional a través de la presentación de un proyecto de ley de urgente consideración ${ }^{24}$, amparado en la Constitución de la República.

Dentro de las áreas que se abordan en el proyecto de ley, se ubica la educación. El principal promotor de los cambios propuestos es el actual Ministro de Educación y Cultura, Pablo Da Silveira, ya mencionado en este texto como uno de los creadores de la Fundación Impulso.

Si bien un abordaje detallado del proyecto excede los alcances del presente artículo, es necesario señalar como contextualización general que el mismo introduce cambios en 57 artículos de la actual Ley General de Educación $\mathrm{N}^{\mathrm{o}}$ 18.437 (URUGUAY, 2008), a la vez que deroga otros 12.

El proyecto de ley innova en tres temas que muestran formas de promoción de la privatización. Estos tienen que ver con la concepción general de educación, la formación docente y el Estatuto que regula el trabajo de los funcionarios docentes y no docentes.

Con respecto al primer elemento, se elimina la potestad de la Comisión Coordinadora de la Educación Pública (conformada por autoridades de la

22 Ver la entrevista disponible en: https://www.elpais.com.uy/informacion/iniciativa-crearliceos-horario-extendido.html.

23 Ver: https://lacallepou.uy/compromiso.pdf.

24 Proyecto disponible en: https://parlamento.gub.uy/documentosyleyes/fichaasunto/145885/ficha_completa 
educación púbica) de planificar la educación pública, según dicta la ley 18437. En su lugar, se propone instalar una Comisión que incorpora a actores de la educación privada en la planificación de la educación pública.

En segundo lugar, se aborda la formación de los docentes. Actualmente la formación de docentes pública en Uruguay es terciaria no universitaria. Se organiza en treinta y tres centros distribuidos en todo el país. La Ley vigente señala que el sistema de formación docente debe transitar hacia modalidades universitarias. El proyecto del gobierno, en su artículo 197, elimina este aspecto, estableciendo un sistema en el cual cada centro público podrá postular para ser reconocido como de universitario por el Ministerio de Educación y Cultura. Así, se coloca a cada centro en competencia con los otros por el acceso al grado universitario.

A su vez, es necesario señalar que existen en Uruguay actualmente cinco centros privados de formación de docentes, tres de ellos pertenecen a universidades privadas. La propuesta del nuevo gobierno incorpora, en su artículo 173, la conformación de un sistema de becas al cual podrían aspirar estudiantes que cursen su formación en entidades universitarias. Por tanto, se trata de becas concebidos exclusivamente para instituciones privadas.

El tercer aspecto a tener en cuenta es la propuesta de establecer, en algunos centros educativos, un nuevo estatuto que regule el trabajo docente. Esta propuesta, incluida en el artículo 191, incorpora dos elementos que resultan particularmente relevantes para el enfoque de trabajo del presente texto. Por una parte se plantea que se podrán establecer,

Compensaciones o complementos salariales y otros beneficios, atendiendo a circunstancias como la ubicación geográfica del lugar de trabajo, el contexto socio-cultural en el que funciona un establecimiento, o el cumplimiento de metas de política pública establecidas en cada caso, de acuerdo con las atribuciones legales y constitucionales establecidas al efecto (URUGUAY, 2020).

Las dos primeras causales señaladas están presentes en la la educación uruguaya. La tercera, es novedosa y configura una forma de privatización endógena (BALL; YOUDELL, 2008).

Por otra parte, la propuesta también prevé que puedan delegarse a los directores de los centros competencias vinculadas a "la conformación de planteles estables, con permanencia de funcionarios y concentración de carga horaria en un mismo centro educativo" (URUGUAY, 2020). Para ello los 
directores podrán tener en cuenta "condiciones de orden funcional (como el compromiso con una metodología de trabajo o un proyecto de centro) para el acceso o permanencia en un lugar de trabajo específico" (URUGUAY, 2020). Esta formulación altera el ordenamiento legal vigente, el cual establece garantías para acceso y permanencia de los docentes en su lugar de trabajo.

Estos cambios en la normativa legal innovan en el sentido de crear un conjunto de centros educativos organizados con lógicas de gestión ya implementadas en el Liceo Impulso. Comienza así a cerrarse un proceso de progresiva privatización de la educación uruguaya.

Estas modalidades de privatización se enmarcan en la modalidad que Ball y Youdell (2008) han denominado como privatización endógena, la cual se caracteriza por "la importación de ideas, técnicas y prácticas del sector privado a fin de tornar al sector público más parecido a la actividad del negocio privado" (BALL; YOUDELL, 2008, p. 8).

\section{Breves conclusiones como parte de un trabajo en curso}

Los principales argumentos abordados en este texto pueden sintetizarse en cinco puntos:

1. Durante los gobiernos progresistas en Uruguay (2005-2019) se desarrollaron procesos de "privatización latente". Estos fueron promovidos por sectores de derecha y tuvieron condiciones para su consolidación debido a la ausencia de debate político-pedagógico por parte de la izquierda. Ello puede deberse a que las fuerzas progresistas asumieron una visión consensual de la democracia, rehuyendo su carácter antagónico. Los procesos de privatización articularon modalidades exógenas y endógenas, con una primacía de estas últimas.

2. El instrumento de las "donaciones especiales" se convirtió en el canal prioritario de financiamiento de experiencias educativas funcionales a los procesos de privatización.

3. Dentro de estas experiencias educativas, el Liceo Impulso asume un lugar privilegiado. Este cumple una función prioritaria en una doble estrategia discursiva:

a) la que se genera a partir de la instalación de la idea de "crisis de la educación" como definición de la realidad uruguaya;

b) la construcción de la "educación pública de gestión privada" como alternativa a la pública. 
4. El capital transnacional y sus manifestaciones locales ocupan un lugar central en el proceso. La articulación empresarial que se genera en apoyo a la iniciativa privatizadora resulta sumamente efectiva para lograr movilizar recursos públicos hacia los fines deseados.

5. La instalación de un nuevo gobierno a partir de marzo del 2020 ha generado las condiciones para la profundización de los procesos de privatización. El nuevo gobierno reactualiza la estrategia iniciada previamente y la relanza como base de una política educativa nacional.

Entendemos que estos cinco puntos resumen la argumentación presentada en el presente artículo. Para una correcta interpretación del conjunto del material expuesto, y de las posibilidades de configuración a futuro de las relaciones entre público y privado en Uruguay, es necesario volver a subrayar nuestra perspectiva acerca del carácter no determinado de los procesos sociales. El hecho que estemos ante un proceso de imposición de procesos de privatización no implica en modo alguno que estos vayan a producirse tal como son promovidos por quienes los impulsan. El modo en que los vínculos entre público - privado se vayan consolidando tendrá que ver con disputas que se han de dar en un campo indecible a priori. Una buena dosis de lo que ha de suceder tendrá que ver con si se establece una frontera entre partidarios de la privatización y defensores del carácter rector del Estado como garante del derecho a la educación, organizándose un espacio de antagonismo y de disputas por la hegemonía. La continuidad de nuestro trabajo investigación permitirá producir textos que den cuenta de nuevas dimensiones de los procesos abordados en el presente artículo, así como de las formas en que las disputas se vayan organizando.

\section{REFERENCIAS}

ADRIÃO, Theresa; PERONI, Vera Maria Vidal. Público e privado na educação: novos elementos para o debate. São Paulo: Xamã, 2008.

BALL, Stephen J. Política social y educativa, empresa social, hibridación y nuevas comunidades discursivas. Propuesta Educativa, Buenos Aires, n. 36, año 20, v. 2, p. 25-34, 2011.

BALL, Stephen J. Globalización, mercantilización y privatización: tendencias internacionales en Educación y Política Educativa. Archivos Analíticos de Políticas Educativas, Arizona, v. 22, n. 41, 2014.

BALL, Stephen J.; YOUDELL, Deborah. Hidden privatisation in public education. Brussels: Education International, 2008. 
BELLEI, Cristián; ORELLANA, Victor. What Does “Education Privatisation” Mean? Conceptual Discussion and Empirical Review of Latin American Cases (ESP Working Paper Series - Open Society Foundations No. 62). New York: Open Society Foundations, 2014.

BORDOLI, Eloísa et al. Privatización educativa en Uruguay: políticas, actores y posiciones. Montevideo: Internacional de la Educación, 2017.

BORDOLI, Eloísa; ROMANO, Antonio. Introducción. En: ROMANO, Antonio; BORDOLI, Eloísa (org.). Pensar la escuela como proyecto [político] pedagógico. Montevideo: PsicoLibros Waslala, 2009, p. 15-22.

CAETANO, Maria Raquel. O protagonismo do setor privado na reforma do Ensino Médio no Brasil: o Instituto Unibanco e suas relações. En: PERONI, Vera Maria Vidal; IMA, Paula Valim de; KADER, Carolina Rosa (org.). Redefinições das fronteiras entre o público e o privado. Implicações para a democratização da educação. São Leopoldo: Oikos, 2018, p. 147-158.

DUFRECHOU, Hugo et al. El avance privatizador en la educación uruguaya: discursos y políticas. Montevideo: Internacional de la Educación, 2019.

LACLAU, Ernesto. La imposibilidad de la sociedad. En: LACLAU, Ernesto. Nuevas reflexiones sobre la revolución de nuestro tiempo. Buenos Aires: Verso, 1993, p. 103-107.

LACLAU, Ernesto; MOUFFE, Chantal. Hegemonía y estrategia socialista. Hacia una radicalización de la democracia. Buenos Aires: Fondo de Cultura Económica, 2004.

MARTINIS, Pablo. Modelos educativos privatizadores y disputas por el derecho a la educación. En: SERPAJ. Derechos humanos en el Uruguay. Informe 2019. Montevideo: Tradinco, 201, p. 323-332.

MINISTERIO DE EDUCACIÓN Y CULTURA (MEC). Logro y nivel educativo de la población. Montevideo: MEC, 2018.

MOSCHETTI, Mauro et al. The increasing role of non-State actors in education policymaking. Evidence from Uruguay. Journal of Education Policy, [s.l.], v. 34, n. 3, p. 367-393, 2019.

MOUFFE, Chantal. En torno a lo político. Buenos Aires: FCE, 2009.

PARTIDO COLORADO (2019). Programa de Gobierno. Montevideo, 2019 Disponible en: https://partidocolorado.uy/un-pequeño-pais-modelo-programa-degobierno-2020-2025/. Acesso en: 14 mayo 2020

PERONI, Vera Maria Vidal. Redefinições das fronteiras entre o público e o privado: implicações para a democratização da educação. Brasilia: Liber Livro, 2013.

STEINER-KHAMSI, Gita. The Politics of League Tables. Journal of Social Science Education, [s.l.], v. 2, n. 1, 2003. 
URUGUAY. Ministerio de Educación y Cultura. Ley No 18.437 de 12 de diciembre de 2008. Ley General de Educación. Diario Oficial, 16 ene/009 - № 27654, Montevideo, 2008. Disponible en: https://www.ineed.edu.uy/images/pdf/-18437-ley-general-deeducacion.pdf. Acesso en: 20 mayo 2020.

URUGUAY. Ministerio de Economía y Finanzas. Ley No 18.083, 27 dic. 2006. Sistema tributario.se derogan, crean y modifican diversas normas. Diario Oficial, 18 ene/007 - No 27163. Montevideo, 2007. Disponible en:https://legislativo.parlamento.gub.uy/ temporales/leytemp4818878.htm. Acesso en: 21 mayo 2020.

URUGUAY. Proyecto de Ley con rótulo de urgente consideración. Montevideo, 2020. Disponible en: https://parlamento.gub.uy/documentosyleyes/ficha-asunto/145885.Acceso en: 22 mayo 2020.

VERGER, Antoni; FONTDEVILA, Clara; ZANCAJO, Adrián. The privatisation of education: a political economy of global education reform. New York: Teachers College Press, 2016.

VERGER, Antoni; MOSCHETTI, Mauro; FONTDEVILA, Clara. La privatización educativa en América Latina: Una cartografía de políticas, tendencias y trayectorias. Bruselas: Internacional de la Educación, 2017.

\section{Fuentes consultadas}

Caras y Caretas (Semanario). Edición del 30 de junio de 2018. Artículo: La telaraña de Techint y Tenaris. Disponible en: https://www.carasycaretas.com.uy/la-telarana-detechint-y-tenaris/

Compromiso por el País (2019). Documento programático de respaldo a la candidatura de Luis Lacalle Pou. Disponible en: https://lacallepou.uy/compromiso.pdf. Acceso en: 14 mayo 2020.

Ernesto Talvi, sitio web de su candidatura. Disponible en: https://talvi.uy.

Diario El País. Una iniciativa para crear 136 liceos con un horario extendido. Montevideo, 12 de junio de 2016.

Liceo Impulso. Disponible en: http://www.liceoimpulso.edu.uy/fundacin

Ministerio de Economía y Finanzas (MEF) (2014). Rendición de cuentas y balance de ejecución presupuestal. Ejercicio 2013. Anexo "Donaciones especiales". Mimeo.

Ministerio de Economía y Finanzas (MEF) (2015). Rendición de cuentas y balance de ejecución presupuestal. Ejercicio 2014. Anexo "Donaciones especiales". Mimeo.

Ministerio de Economía y Finanzas (MEF) (2016). Rendición de cuentas y balance de ejecución presupuestal. Ejercicio 2015. Anexo “Donaciones especiales”. Disponible en: https://www.gub.uy/ministerio-economia-finanzas/rendicion-cuentas-2015. Disponible en: 21 mayo de 2020 . 
Ministerio de Economía y Finanzas (MEF) (2017). Rendición de Cuentas y Balance de Ejecución Presupuestal 2016. Análisis Presupuestal. Disponible en: https://www.gub.uy/ ministerio-economia-finanzas/rendicion-cuentas-2017. Disponible en: 21 de mayo 2020.

Ministerio de Economía y Finanzas (MEF) (2018). Rendición de Cuentas y Balance de Ejecución Presupuestal 2017. Análisis Presupuestal. Disponible en: https://www.gub.uy/ ministerio-economia-finanzas/rendicion-cuentas-2017. Acesso en: 21 de mayo 2020.

Ministerio de Economía y Finanzas (MEF) (2019). Rendición de cuentas y balance de ejecución presupuestal. Ejercicio 2018. Exposición de motivos. Anexo 4.5 "Donaciones especiales con exoneraciones fiscales. Disponible en: https://www.gub.uy/ministerioeconomia-finanzas/tramites-y-servicios/contenido/presupuesto/rendicion-cuentas-2018balance-ejecucion-presupuestal. Disponible en: 21 mayo 2020.

Ministerio de Educación y Cultura (MEC) (2008). Ley General de Educación. Montevideo: Impresiones y Publicaciones Oficiales (IMPO).

Uruguay (1967). Constitución de 1967 con las modificaciones plebiscitadas el 26 de noviembre de 1989, el 26 de noviembre de 1994, el 8 de diciembre de 1996 y el 31 de octubre de 2004. Disponible en: https://www.impo.com.uy/bases/constitucion/1967-1967.

Texto recibido en $28 / 07 / 2020$.

Texto aprobado en 14/09/2020

Este é um artigo de acesso aberto distribuído nos termos de licença Creative Commons.

(cc) BY 